\title{
Limited value of KAI1/CD82 protein expression as a prognostic marker in human gastric cancer
}

\author{
Maximilian Knoener, Till Krech, Florian Puls, Ulrich Lehmann, Hans Kreipe and Matthias Christgen* \\ Institute of Pathology, Hannover Medical School, Hannover, Germany
}

\begin{abstract}
The cell surface glycoprotein KAI1/CD82 suppresses tumor growth and metastasis in animal models. This study aimed to evaluate the prognostic relevance of KAI1/CD82 protein expression in human gastric cancer. Primary gastric carcinomas ( $n=271)$ with a mean clinical follow-up time of 48 months were immunostained using the monoclonal anti-KAI1/CD82 antibody G2. Staining was evaluated as negative versus positive for statistical analysis. KAI1/CD82 immunoreactivity was absent in 103/271 (38\%) cases. There was a trend towards KAI1/CD82 negativity in poorly differentiated cases $(p=0.0679)$. Moreover, KAI1/CD82-negative carcinomas were associated with a higher $\mathrm{pT}$ status $(p=0.0222)$, metastatic lymph node involvement $(p=0.0018)$ and a higher clinical tumor stage $(p=0.0050)$. The median overall survival times of KAI1/CD82-negative and KAI1/CD82-positive gastric carcinomas were 20 and 37 months, respectively $(p=0.2305)$. These results are in line with the proposed function of KAI1/CD82 as a suppressor of tumor growth and metastasis. However, these data suggest that KAI1/CD82, as detected by immunohistochemistry, is of limited value as a prognostic marker for gastric cancer in routine histological workup.
\end{abstract}

Keywords: Gastric cancer, prognostic markers, metastasis, immunohistochemistry

\section{Introduction}

KAI1/CD82, subsequently referred to as KAI1, belongs to the tetraspanin superfamily of cell surface glycoproteins, which are implicated in the regulation of tumor growth and progression. Originally, KAI1 was identified as a suppressor of metastasis in experimental animal models [1]. Ectopic expression of KAI1 inhibits tumor growth and metastasis of highly aggressive tumor cell lines, including AT6.1 and LNCaP prostate cancer cells and B16BL6 melanoma cells [14]. KAI1 interacts with the Duffy antigen receptor for chemokines (DARC), which is expressed on vascular endothelial cells and on lymphatic vessels [3]. This interaction transmits a senescent signal to intravasated

${ }^{*}$ Corresponding author: Matthias Christgen, Institute of Pathology, Hannover Medical School, 30625 Hannover, Germany. Tel.: +49 511532 4501; Fax: +49 511532 4488, E-mail: Christgen. Matthias@MH-Hannover.de. tumor cells expressing KAI1, whereas KAI1-negative cancer cells continue to proliferate and ultimately give rise to metastases [3]. Therefore, KAI1 has been proposed as a promising new biomarker for predicting metastatic spread and prognosis in a wide variety of human malignancies. Analyses of clinical tumor specimens revealed that the expression of KAI1 is frequently downregulated or lost in several types of carcinomas [5-9]. In prostate cancer for instance, expression of KAI1 is downregulated or lost in poorly differentiated cases $[5,6]$. However, with respect to gastric cancer, the clinical relevance of KAI1 expression remained controversial. Guo and colleagues reported that KAI1 expression was not altered or lost in a series of 35 gastric cancers [10]. In contrast, Tsutsumi et al. observed a strong KAI1 immunoreactivity in the normal gastric epithelium, but a loss of KAI1 protein expression in a considerable proportion of gastric cancers [11]. In the tumor cohort investigated by Tsutsumi and colleagues, KAI1-negative cases were associated with a higher nodal stage and with shortened patient 
Table 1

Characteristics of the tumor set

\begin{tabular}{|c|c|c|c|c|c|}
\hline & Number & Percentage & & Number & Percentage \\
\hline Cases & 271 & 100 & Borrmann classification & & \\
\hline Age & & & type I & 23 & 9 \\
\hline males & & & type II & 109 & 40 \\
\hline$<70$ years & 127 & 47 & type III & 74 & 27 \\
\hline$\geqslant 70$ years & 48 & 18 & type IV & 33 & 12 \\
\hline females & & & not specified & 32 & 12 \\
\hline$<75$ years & 71 & 26 & Tumor localization $^{*}$ & & \\
\hline$\geqslant 75$ years & 25 & 9 & esophagogastric junction & 73 & $27^{*}$ \\
\hline Sex & & & cardia & 116 & $43^{*}$ \\
\hline male & 175 & 65 & corpus & 91 & $34^{*}$ \\
\hline female & 96 & 35 & pylorus & 91 & $34^{*}$ \\
\hline pT status & & 35 & pyloroduodenal junction & 4 & $1^{*}$ \\
\hline pT1 & 34 & 12 & entire stomach & 1 & $<1^{*}$ \\
\hline pT2 & 127 & 12 & not specified & 17 & $6^{*}$ \\
\hline pT3 & 92 & 41 & Histology & & \\
\hline pT4 & 18 & 34 & adenocarcinoma, NOS & 130 & 47 \\
\hline$p N$ status & 10 & 7 & adenocarcinoma, intestinal type & 25 & 9 \\
\hline & & & adenocarcinoma, diffuse type & 4 & 2 \\
\hline $\mathrm{pNO}$ & 86 & 31 & papillary adenocarcinoma & 2 & 1 \\
\hline $\mathrm{pN} 1$ & 91 & 34 & tubular adenocarcinoma & 29 & 11 \\
\hline $\mathrm{pN} 2$ & 91 & 34 & mucinous adenocarcinoma & 6 & 2 \\
\hline $\mathrm{pN} 3$ & 3 & 1 & signet-ring cell carcinoma & 53 & 19 \\
\hline pM status & & & adenosquamous carcinoma & 1 & 1 \\
\hline pM0 & 243 & 90 & undifferentiated carcinoma & 3 & 1 \\
\hline pM1 & 28 & 10 & others and mixed histology & 2 & 1 \\
\hline Histological grade & & & not specified & 16 & 6 \\
\hline G1 & 9 & 3 & Karnofsky index & & \\
\hline $\mathrm{G} 2$ & 135 & 50 & $<80$ & 24 & 9 \\
\hline G3 & 127 & 47 & $\geqslant 80$ & 125 & 46 \\
\hline Laurén classification & & & unspecified & 122 & 45 \\
\hline intestinal type & 154 & 57 & Clinical stage & & \\
\hline diffuse type & 49 & 18 & IA & 29 & 11 \\
\hline mixed type & 24 & 9 & IB & 46 & 17 \\
\hline not specified & 44 & 16 & II & 56 & 20 \\
\hline Ming classification & & & IIIA & 59 & 22 \\
\hline expansive type & 141 & 52 & IIIB & 40 & 15 \\
\hline infiltrative type & 55 & 20 & Survival status & 41 & 15 \\
\hline not specified & 75 & 28 & Survival status & & \\
\hline & & & deceased & 170 & 63 \\
\hline
\end{tabular}

overall survival [11]. Similar findings were also reported by Lee et al. and Wu et al. in two other gastric cancer cohorts $[12,13]$. The present study aimed to evaluate the clinical relevance of KAI1 protein expression in the largest series of gastric carcinomas with clinical follow-up data analyzed for KAI1 expression reported in the literature so far.

\section{Materials and methods}

\subsection{Patients}

The present work included primary gastric cancer specimens of 271 patients who had participated in a clinical study conducted at the Hannover Medical School between 1986 and 1997 [14]. Inclusion criteria of this study had comprised complete surgical R0 resection of the primary tumor, histologically proven gastric carcinoma, no neoadjuvant or adjuvant chemotherapy or radiotherapy and absence of secondary malignancies. All patients gave their informed consent and the study was approved by the local ethics committee of Hannover Medical School in compliance with the code of ethics of the World Medical Association (Declaration of Helsinki). The clinicopathological characteristics of the tumors are shown in Table 1. Macroscopic tumor appearance was evaluated according to the Borrmann classification and also considered the tumor localization within the stomach [15]. Tumor histology was determined according to the criteria of the World Health Organization and according to the Laurén and the Ming classifications [16-18]. The pTNM stage and the clinical tumor stage were assessed according to the unified 
international gastric cancer staging classification system, as incorporated in the American Joint Committee on Cancer (AJCC) manual for staging of cancer, 3rd edition, and in the Union Internationale Contre le Cancer (UICC) TNM classification manual, 4th edition [19-21]. Clinical follow-up data including overall survival were available for all patients. The mean clinical follow-up time was 48 months (4 years, range: 1163 months). One-hundred and seventy patients (63\%) died during the observation time.

\subsection{Tissue microarrays and immunohistochemistry}

Tissue microarrays containing $1.4 \mathrm{~mm}$ (diameter) core biopsies from representative tumor areas of formalin-fixed paraffin-embedded gastric cancer tissue were constructed as described previously [14,22]. For KAI1 immunohistochemistry, $4 \mu \mathrm{m}$ sections of tissue microarrays were mounted on poly- ${ }_{L}$-lysine coated slides. Slides were deparaffinized and rehydrated conventionally. Antigen retrieval was achieved by pressure cooking at $125^{\circ} \mathrm{C}$ in $10 \mathrm{mmol} / \mathrm{l}$ citric acid (pH 6) for $3 \mathrm{~min}$. Endogenous peroxidase was blocked with $0.3 \%$ (v/v) $\mathrm{H}_{2} \mathrm{O}_{2}$ and slides were subjected to immunohistochemical staining using the monoclonal anti-KAI1 antibody G2 (Santa Cruz Biotechnology, Santa Cruz, CA, U.S.A) as described previously [8]. The ZytoChemPlus HRP Kit (Zytomed, Berlin, Germany) was used for detection of the immune reaction. The UACC-893 and MCF-7 cell lines were employed as internal positive and negative controls in each staining batch as described previously [8]. Evaluation of membranous KAI1 immunoreactivity was performed using an immunoreactivity score (IRS) as described by Remmele and Stegner [23]. In brief, IRS was calculated as the product of staining intensity (graded between negative $=0$ and strong $=3$ ) and the percentage of positively stained tumor cells (graded between 0 and 4 , being $1<25 \%, 2=25-50 \%, 3=51-75 \%, 4>75 \%$ ). Tumors with an IRS $\leqslant 2$ were considered as KAI1negative, whereas those with an IRS $\geqslant 3$ were considered as KAI1-positive. All tissue microarrays were scored independently by four observers (MC, MK, TK and FP) and divergent scorings were discussed and decided on a multi-head microscope.

\subsection{Statistics}

Statistical analyses were performed with GraphPad Prism software. The $\chi^{2}$ test and the $\chi^{2}$ test for trends were used to assess the statistical significance of asso- ciations between KAI1 expression status and clinicopathological parameters. Univariate survival analyses were performed by the Kaplan-Meier method. Differences in the survival curves were assessed by log-rank test. $P$ values $<0.05$ were considered significant.

\section{Results}

A collection of 271 primary gastric carcinomas were compiled onto tissue microarrays and immunostained for KAI1. Absence of KAI1 immunoreactivity was observed in 103/271 (38\%) cases (Table 2 and Fig. 1D). In KAI1-negative versus KAI1-positive gastric carcinomas, there was no significant difference with respect to gender distribution, patient age, Karnofsky index, presence of distant metastasis at the time of diagnosis, macroscopic tumor appearance, tumor localization and histological classification (Table 2). There was a trend towards KAI1 negativity in poorly differentiated cases $(p=0.0679)$ (Table 2$)$. However, this was not statistically significant. Instead, KAI1-negative carcinomas were significantly associated with a higher pT status $(p=0.0222)$ and a higher $\mathrm{pN}$ status $(p=$ 0.0018) (Table 2). Consistently, KAI1-negative carcinomas were also associated with a higher clinical tumor stage $(p=0.0050)$ (Table 2$)$. Moreover, KAI1negative carcinomas were over-represented in a subset of patients who died early ( $<27$ months) after tumor diagnosis (Table 2). This prompted us to determine whether loss of KAI1 expression was associated with shortened patient overall survival, when taking into consideration the entire clinical follow-up data. In univariate survival analyses, cumulative survival curves were calculated according to the Kaplan-Meier method. Differences in survival were assessed with the log-rank test. Conventional prognostic markers used in routine clinical pathology, such as pT status, lymph node involvement and presence of distant metastases reached significance for overall survival in the tumor collection analyzed (Figs $1 \mathrm{~A}-\mathrm{C}$ ). For instance, the median overall survival times of pT4 and pT2 tumors were 10 and 40 months, respectively ( 0.8 and 3.3 years, $p<0.0001$ ) (Fig. 1A). The median overall survival times of KAI1-negative and KAI1-positive cases were 20 and 37 months, respectively (1.7 and 3.1 years, $p=$ 0.2305) (Fig. 1E). Thus, KAI1-negative gastric carcinomas were not statistically significantly associated with shortened patient overall survival. 
Table 2

Relationship between KAI1 protein expression and clinicopathological parameters in gastric cancer

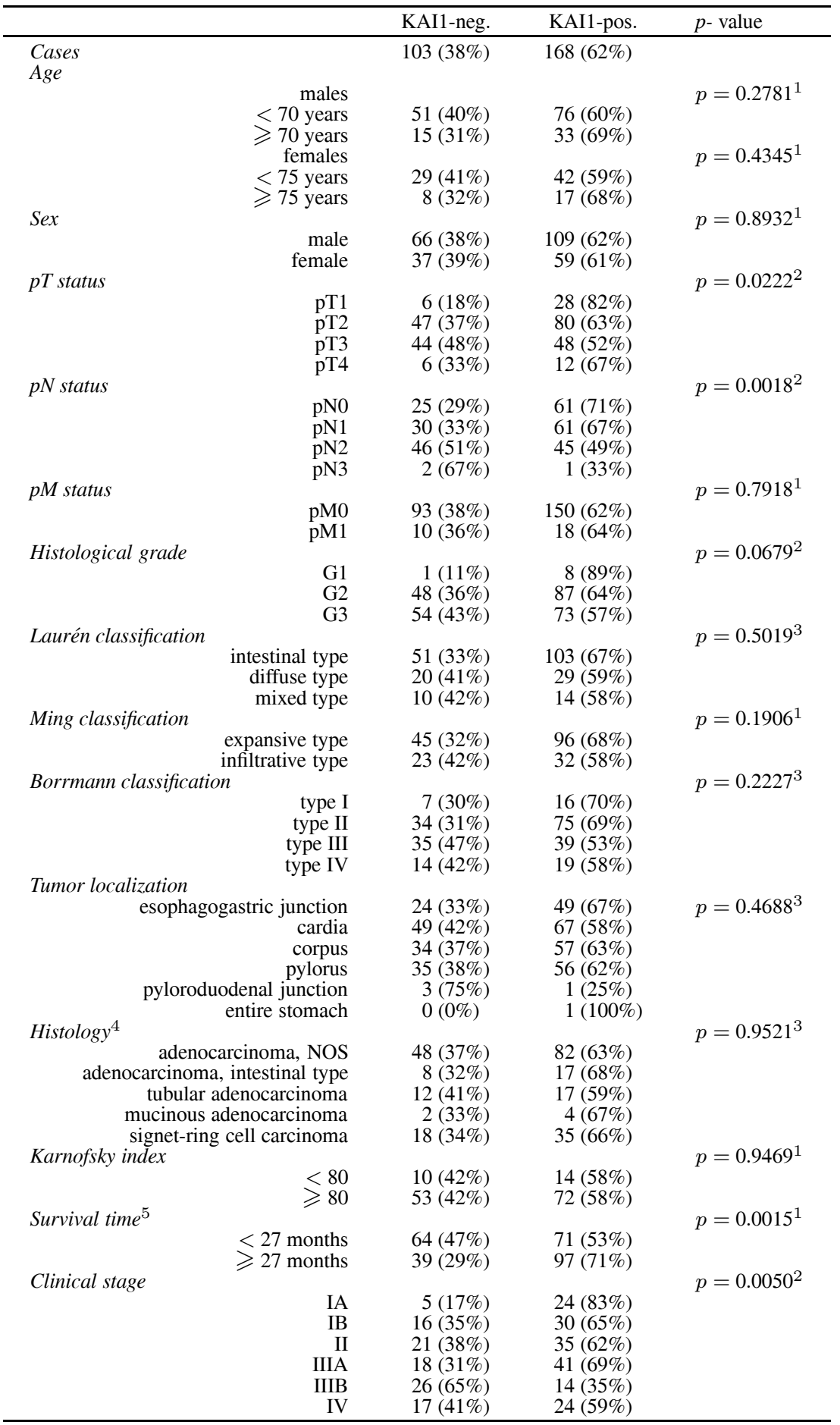

${ }^{1} \chi^{2}$ test.

${ }^{2} \chi^{2}$ test for trends.

${ }^{3} \chi^{2}$ test for independence.

4 the most prevalent histological subtypes were included.

${ }^{5} 27$ months corresponds to the median survival time of all the patients in the study. 


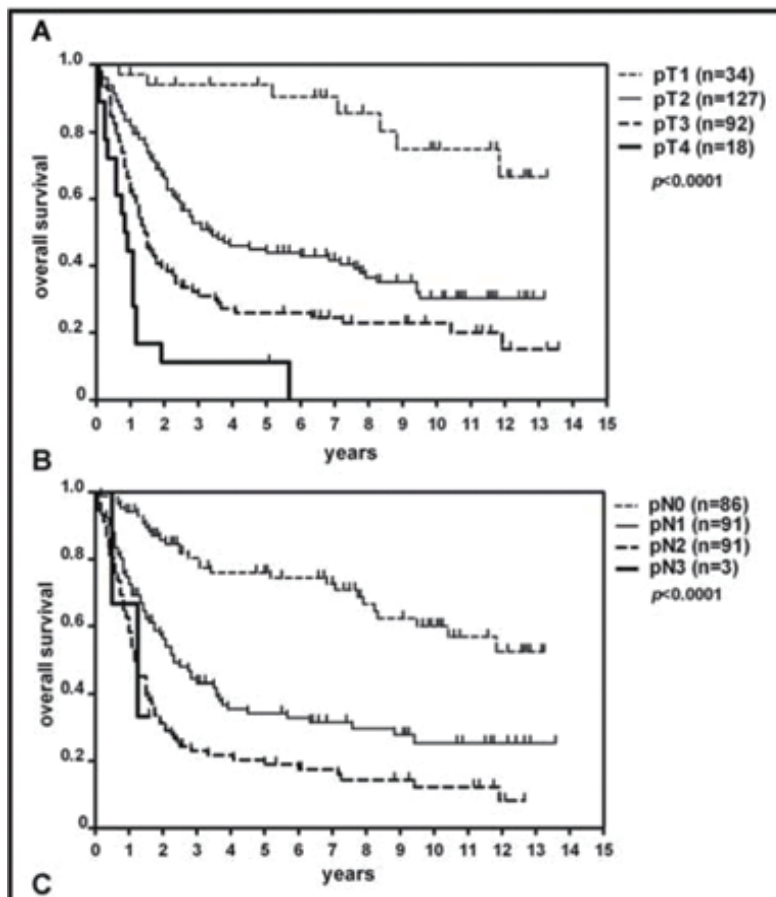

\section{D}
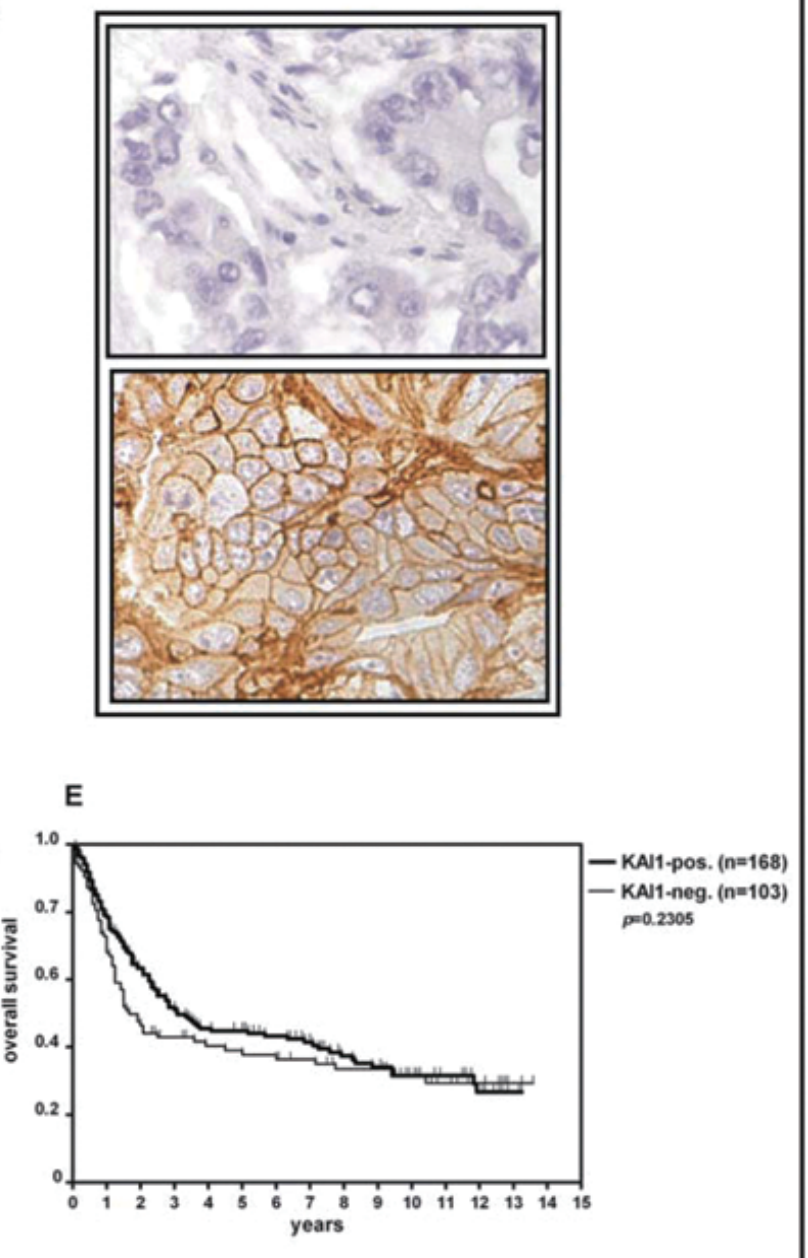

Fig. 1. Patient overall survival dependent on the pT stage (A), the pN stage (B), the pM stage (C) and the expression of KAI1 (E). Censored individuals are indicated by vertical lines. KAI1 immunohistochemical stainings representative of gastric carcinomas scored as KAI1-negative or KAI1-positive are shown in the upper right panel (D).

\section{Discussion}

KAI1 suppresses tumor growth and metastasis in experimental animal models and is frequently downregulated or lost in various types of human tumors [1-9]. KAI1 has been proposed as a promising new prognostic biomarker for a variety of malignancies. The present study aimed to evaluate the use of KAI1 expression as a prognostic marker in gastric cancer. KAI1 protein expression was determined by immunohistochemistry in the largest series of gastric carcinomas with clinical follow-up data analyzed for KAI1 expression reported in the literature so far. In line with previous findings reported independently by Tsutsumi et al., Lee et al., and $\mathrm{Wu}$ et al., we observed a loss of KAI1 expression in a considerable proportion $(38 \%)$ of primary gastric carcinomas [11-13]. KAI1-negative gastric carcinomas were associated with a higher pT status, a higher $\mathrm{pN}$ status and a higher clinical tumor stage, which is consistent with the proposed function of KAI1 as a suppressor of tumor growth and metastasis. There was a trend towards KAI1 negativity in poorly differentiated gastric carcinomas, which has previously also been observed in prostate carcinomas [5,6]. However, KAI1-negative status was not significantly associated with presence of distant metastasis at the time of tumor 
diagnosis. This may be due to the limited number of patients with a pM1 status in the initial tumor staging included in this study. KAI1-negative gastric carcinomas were over-represented in a subset of patients who died early ( $<27$ months) after tumor diagnosis. However, when taking into consideration the entire clinical follow-up data, KAI1-negative cases were not significantly associated with shorter patient overall survival, as had previously been reported by Tsutsumi et al. and Lee et al. [11,12]. Of note, the present study has been conducted based on gastric cancer samples of a patient cohort that had undergone surgical tumor resection before adjuvant chemoradiotherapy had been established as the standard treatment procedure for gastric cancer [24]. This also applies to the studies of Tsutsumi et al. and Lee et al. [11,12]. On the one hand, we can not exclude that slightly different results might be obtained in more recent patient cohorts, which have received adjuvant chemoradiotherapy. On the other hand, this eliminates potential confounding effects of therapy modalities on the impact of KAI1 on overall survival and facilitates a comparison with the aforementioned studies of Tsutsumi et al. and Lee et al. [11,12]. Collectively, our findings suggest that KAI1, as detected by immunohistochemistry, is of limited clinical value as a prognostic marker for gastric carcinoma in routine histological workup.

\section{References}

[1] J.T. Dong, P.W. Lamb, C.W. Rinker-Schaeffer, J. Vukanovic, T. Ichikawa, et al. KAI1, a metastasis suppressor gene for prostate cancer on human chromosome 11p11.2, Science $\mathbf{2 6 8}$ (1995), 884-6.

[2] J.H. Kim, B. Kim, L. Cai, H.J. Choi, K.A. Ohgi, et al. Transcriptional regulation of a metastasis suppressor gene by Tip60 and beta-catenin complexes, Nature 434 (2005), 921-6.

[3] S. Bandyopadhyay, R. Zhan, A. Chaudhuri, M. Watabe, S.K. Pai, et al. Interaction of KAI1 on tumor cells with DARC on vascular endothelium leads to metastasis suppression, Nat Med 12 (2006), 933-8.

[4] X. Yang, L.L. Wei, C. Tang, R. Slack, S. Mueller, et al. Overexpression of KAI1 suppresses in vitro invasiveness and in vivo metastasis in breast cancer cells, Cancer Res 61 (2001), 5284-8.

[5] T. Bouras, A.G. Frauman. Expression of the prostate cancer metastasis suppressor gene KAI1 in primary prostate cancers: a biphasic relationship with tumour grade, J Pathol 188 (1999), 382-8.

[6] T. Ueda, T. Ichikawa, J. Tamaru, A. Mikata, K. Akakura, et al. Expression of the KAI1 protein in benign prostatic hyperplasia and prostate cancer, Am J Pathol 149 (1996), 1435-40.
[7] X.Z. Guo, H. Friess, F.F. Di Mola, J.M. Heinicke, M. AbouShady, et al. KAI1, a new metastasis suppressor gene, is reduced in metastatic hepatocellular carcinoma, Hepatology 28 (1998), 1481-8.

[8] M. Christgen, H. Bruchhardt, M. Ballmaier, T. Krech, F. Langer, et al. KAI1/CD82 is a novel target of estrogen receptor-mediated gene repression and downregulated in primary human breast cancer, Int J Cancer 123 (2008), 2239-46.

[9] H. Huang, J. Groth, K. Sossey-Alaoui, L. Hawthorn, S. Beall, et al. Aberrant expression of novel and previously described cell membrane markers in human breast cancer cell lines and tumors, Clin Cancer Res 11 (2005), 4357-64.

[10] X.Z. Guo, H. Friess, C. Maurer, P. Berberat, W.H. Tang, et al. KAI1 is unchanged in metastatic and nonmetastatic esophageal and gastric cancers, Cancer Res 58 (1998), 753-8.

[11] S. Tsutsumi, T. Shimura, N. Morinaga, E. Mochiki, T. Asao, et al. Loss of KAI1 expression in gastric cancer, Hepatogastroenterology 52 (2005), 281-4.

[12] H.S. Lee, H.K. Lee, H.S. Kim, H.K. Yang, W.H. Kim. Tumour suppressor gene expression correlates with gastric cancer prognosis, J Pathol 200 (2003), 39-46.

[13] Q. Wu, Y. Ji, M.Q. Zhang, Y.Q. Chen, F. Chen, et al. Role of tumor metastasis suppressor gene KAI1 in digestive tract carcinomas and cancer cells, Cell Tissue Res 314 (2003), 23749.

[14] J. Ruschoff, M. Dietel, G. Baretton, S. Arbogast, A. Walch, et al. HER2 diagnostics in gastric cancer-guideline validation and development of standardized immunohistochemical testing, Virchows Arch 457 (2010), 299-307.

[15] R. Borrmann. Geschwulste des Magens und Duodenums. In: Henke F, Lubarsch O, eds. Handbuch der speziellen pathologischen Anatomie und Histologie Berlin: Springer-Verlag, 1926.

[16] WHO. (2000) Pathology and Genetics of Tumours of the Digestive System. Lyon: IARC Press.

[17] T. Lauren. The two histologic main types of gastric carcinoma, Acta Pathol Microbiol Scand 64 (1965), 34.

[18] S.C. Ming. Gastric carcinoma. A pathobiological classification, Cancer 39 (1977), 2475-85.

[19] B.J. Kennedy. The unified international gastric cancer staging classification system, Scand J Gasteroenterol 198722 (1987), 11-13.

[20] UICC. TNM classification of malignant tumours, 4th ed. Berlin: Springer Verlag, (1987).

[21] AJCC. American Joint Committe on Cancer manual for staging cancer, 3rd ed. Philadelphia: Lippincott, (1988).

[22] M. Mengel, R. von Wasielewski, B. Wiese, T. Rudiger, H.K. Muller-Hermelink, et al. Inter-laboratory and inter-observer reproducibility of immunohistochemical assessment of the Ki67 labelling index in a large multi-centre trial, J Pathol 198 (2002), 292-9.

[23] W. Remmele, H.E. Stegner. [Recommendation for uniform definition of an immunoreactive score (IRS) for immunohistochemical estrogen receptor detection (ER-ICA) in breast cancer tissue], Pathologe 8 (1987), 138-40.

[24] J.S. Macdonald, S.R. Smalley, J. Benedetti, S.A. Hundahl, N.C. Estes, et al. Chemoradiotherapy after surgery compared with surgery alone for adenocarcinoma of the stomach or gastroesophageal junction, $N$ Engl J Med 345 (2001), 725-30. 


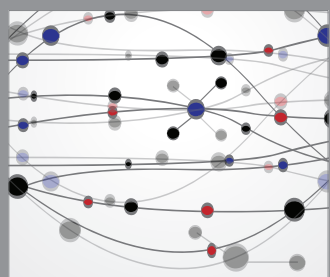

The Scientific World Journal
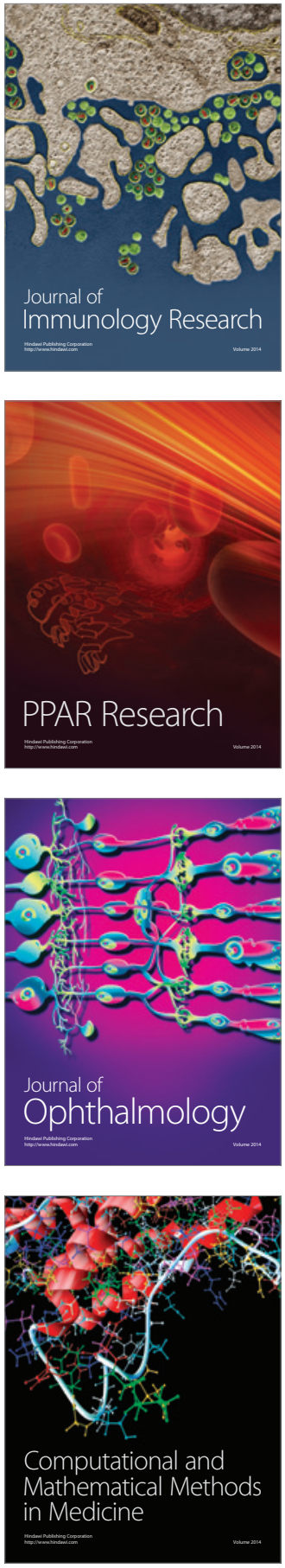

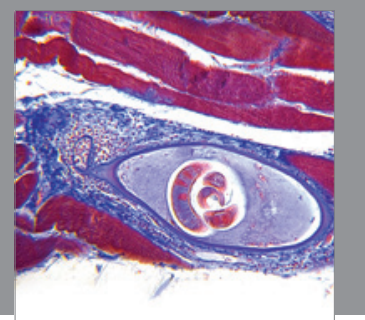

Gastroenterology

Research and Practice
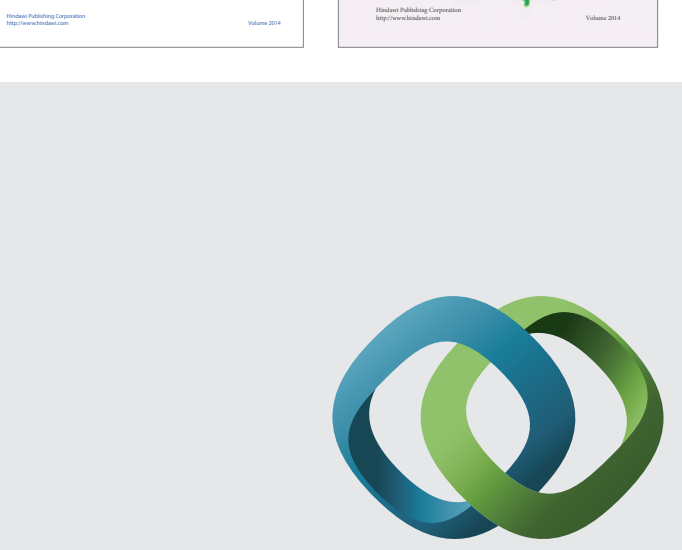

\section{Hindawi}

Submit your manuscripts at

http://www.hindawi.com
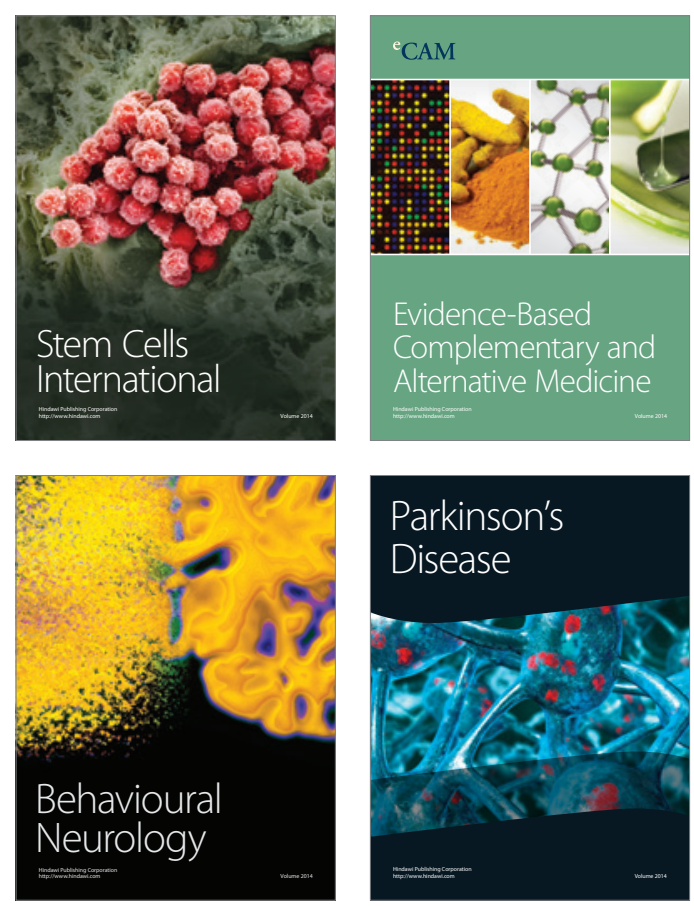

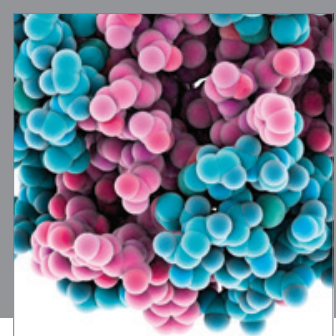

Journal of
Diabetes Research

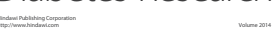

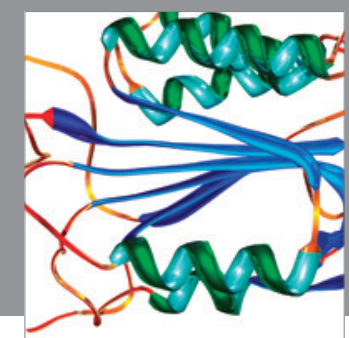

Disease Markers
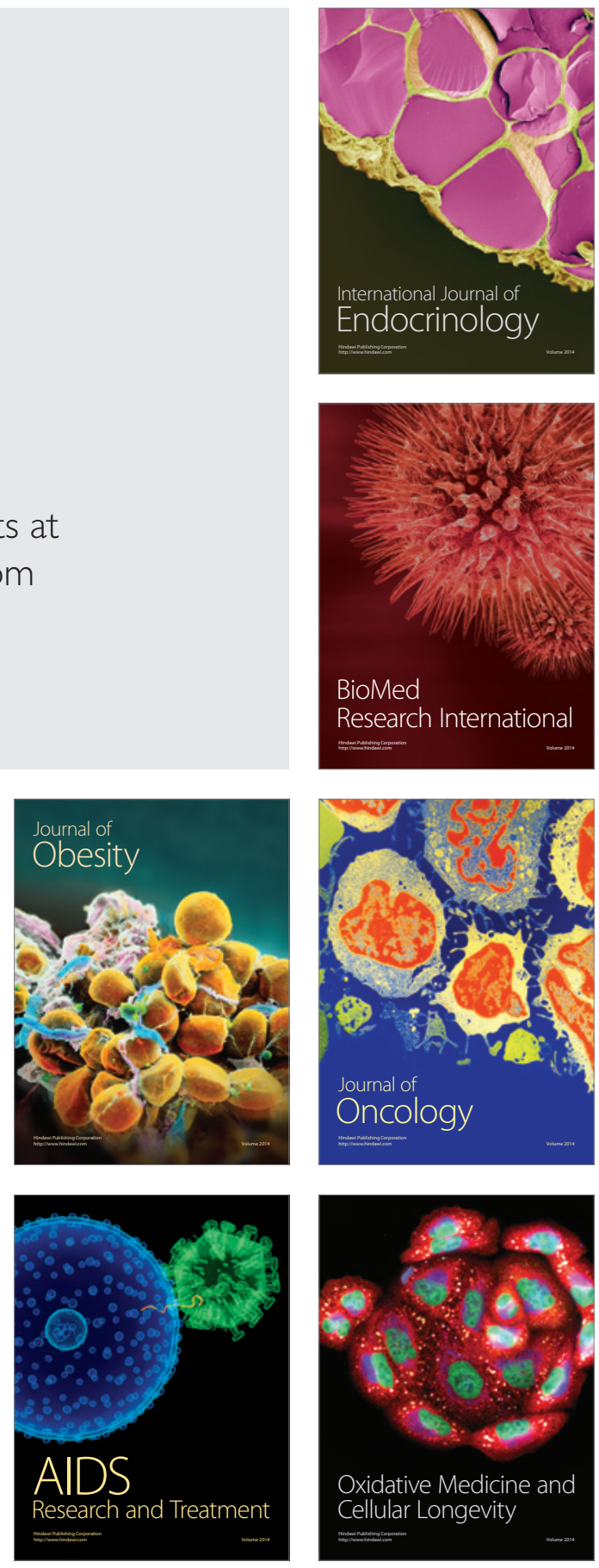